\title{
A METHOD OF CUTTING FROZEN SECTIONS BY EMBEDDING IN GELATIN. ${ }^{1}$
}

\author{
By J. F. Gaskell, Beit Memorial Research Fellow.
}

From the Pathological Laboratory, St. Bartholomew's Hospilal, London, E.C.

METHODS of embedding in gelatin have been made use of by various investigators for special purposes. Usually the sliding microtome has been employed for obtaining sections from the gelatin block. The improvement in the technique of cutting frozen sections, largely due to Aschoff, now enables freezing methods to be easily applied to material embedded in gelatin.

The combination of gelatin embedding and freezing of the block has previously been employed by Hamilton, whose method is described in his textbook published in 1889 (1). Inasmuch as he makes use of a gelatin solution of a strength which is too weak for the method about to be described, that he mixes this with a gum and syrup solution, and that he soaks his tissues for several days, his method differs so considerably from that explained below that further comparison of the two is unnecessary. It may perhaps be mentioned, however, that the present method is found to be very successful with brain material, a tissue which Hamilton says is unsuitable for his methods.

The method has been found particularly useful for obtaining sections of material which it is difficult to cut on a freezing microtome in the ordinary way, either owing to the small size of the objects, or the difficulty of preserving the sections intact when cut. For instance, tissues, such as the spleen and pancreas, give sections which tend to disintegrate into many small fragments, when cut frozen in the ordinary way. The method is also useful in the case of such tissues as the lung in various pathological conditions, for example in bronchopneumonia, as the contents of the bronchi and alveoli are preserved in situ.

It is also claimed that the method has advantages as a routine method, owing to the fact that the avoidance of such fluids as alcohol, xylol, etc., prevents the shrinkage and consequent distortion and vacuolation which occur with paraffin methods: many substances also, such as the fats, which are dissolved by alcohol, are preserved in

1 Received April 27, 1912. 
the section and thus a more faithful picture of the true conditions is obtained.

The most important point in the method is the obtaining of the proper consistency of the gelatin, as it is only when the correct proportion of water is incorporated, that the mass can be satisfactorily manipulated.

The tissues to be embedded can be fixed in various ways, but it has been found that some mixture containing formalin is, on the whole, the most satisfactory; the fixatives most commonly used have been a 10 per cent. solution of formalin (4 per cent. formaldehyde) in isotonic saline solution, or Orth's formol Müller solution, consisting of 10 per cent. formalin in Müller's fluid.

The first point of importance is, that the tissue must be thoroughly well washed before embedding. If any formalin is left in the tissue block, it will formalise the gelatin and prevent penetration of the mass. The routine practice has therefore been to wash out the tissue overnight in a stream of running water. The gelatin employed has been golden label gelatin, as commonly used for the formation of bacterial culture media. It is torn up in pieces and allowed to soak in water for a certain time, depending on the temperature of the laboratory. In England, as a rule, three to four minutes is the proper time; when, however, the method was employed in Naples in June and July, the room temperature being $75^{\circ}-80^{\circ} \mathrm{F}$., any period over one minute was found to be too long. If the gelatin is oversoaked, the resultant mass is not stiff enough to cut easily.

The soaked gelatin is wrung out by hand and is placed in a small beaker, covered to prevent evaporation, and melted. An ordinary paraffin oven will be found convenient for this purpose. The melted gelatin, when of the proper consistency, is rather viscous. It is then transferred to an ordinary incubator at $37^{\circ} \mathrm{C}$, at which temperature the embedding is accomplished. The tissue, thoroughly washed as described above, is freed from superfluous moisture with blottingpaper and then dropped into the fluid gelatin at $37^{\circ} \mathrm{C}$. (never above body temperature), and allowed to soak for at least two hours. Longer periods than four to five hours are unnecessary and are to be avoided, as no better penetration will take place with longer immersions.

The tissue is then cast in paper boxes in the gelatin in which it has been soaked. These boxes are convenient for the further hardening, and can be easily cut away when the block is prepared for cutting.

The mass, allowed to set at room temperature, is then subjected to a hardening process.

The unhardened mass can be frozen and cut, and has the doubtful advantage that the gelatin can be got rid of by soaking in hot water; the sections thus obtained, however, offer no advantages over those obtained frozen in the ordinary way without embedding, as the 
dissolving of the gelatin causes the disintegration of any tissues which will not hold together when cut in the usual manner. No satisfactory method of fixing the section to the slide and then dissolving away the mass has been discovered, as all such methods involve a partial drying of the tissue, which is very injurious to its structure.

The removal of the mass has not been found to be of great importance, as, although it stains, it does not really interfere with the bistological picture. This point will again be referred to when discussing the staining of the sections.

The hardening of the block is done by means of formalin either as vapour or in solution. The vapour method is the more satisfactory. The blocks are placed in a chamber containing strong formalin; a large deep Petri dish is convenient for this. They are supported above the fluid by the fine shavings used for packing, or some similar material. They can then be left in this chamber indefinitely till wanted. Blocks that have been so left for six months have been found to cut easily.

The hardening is not satisfactory in a less period than three days, and improves to some extent with a longer treatment. If the block is not sufficiently hardened, the sections obtained are difficult to manipulate and tend to adhere to the lifter and to each other; when properly hardened they can be manipulated with great ease. If it is required to store the material for a long time, a formalin solution is perhaps the more convenient, a 5 per cent. solution being found satisfactory; it is as well, however, to transfer these blocks to the formalin vapour chamber for a few days before cutting them.

The blocks pared down with a knife, and the paper cut away, are then ready to be sectioned. This paring should be done just before cutting, as the surface of the block dries up to some extent in a short time. The pared block is then put into water for a minute or two preliminary to being frozen. If the block has been long hardened it sometimes does not cut well, the gelatin being rather brittle; this can be remedied by leaving it in water for ten minutes or so; the gelatin takes up some water and becomes less brittle.

The freezing microtome used is the model designed by Professor Aschoff and made by Sartorius in Göttingen. The principle is described in Gaylord and Aschoff"s "Course of Pathological Histology" $\left(1901^{3}\right)$. Carbon dioxide is used as the freezing medium. The water which is always present in the $\mathrm{CO}_{2}$ cylinders often causes serious inconvenience by freezing up in the apparatus. This can be obviated by inserting the cylinder in an upright stand with the feeding nozzle downwards. In twenty-four hours the water will all have collected to the bottom and can be blown out before connecting up with the microtome.

It is somewhat difficult to get a good contact between the gelatin block and the stage of the freezing microtome. The difficulty can be removed by first freezing the block the wrong way up, and cutting a 
smooth surface to be later applied to the stage, and then by applying a drop of gum solution; the smooth side of the block will then freeze firmly on to the stage. Each section is removed from the knife as it is cut and is floated out on a dish of cold water.

The routine thickness worked with is $10 \mu ; 5 \mu$ sections can, however, be obtained with practically all tissues so far tried; for brain they are easily obtained and are necessary for satisfactory results.

Serial sections may be obtained by a method invented by Professor Aschoff.

A large rectangular glass dish has a movable cover, into which are inserted a number of small tubes with a perforation in their base and a loose perforated porcelain plate resting on the bottom of them. The tubes, therefore, can be removed from any fluid in the glass dish by removing the cover, and can be allowed to drain, the sections placed in them being caught on the porcelain plates. The whole can be shifted into any staining or other fluid contained in a similar dish. The fluid wells up into the tubes through their perforated base. Serial sections can thus be simultaneously and equally stained without disarranging them.

As regards staining methods all ordinary watery stains can be used; hæmatoxylin stains are, however, usually preferable to carmine stains. Small crystallising basins will be found much more convenient than watch glasses to stain in; a drawn-out glass rod bent at a right angle is the most convenient form of section lifter. The stains which give the best results are Mayer's acid hæmalum, and Ehrlich's acid hæmatoxylin. The former stain can in great part be removed from the gelatin by treatment for a short time with a 10 per cent. alum solution; the nuclear structures meanwhile retain the stain.

A solution of 10 per cent. watery eosin is useful as a counterstain, the section should be counterstained for about ten minutes, and then thoroughly washed out with water before mounting. If the washing is thorough no diffusion of eosin will take place in the mounting medium.

It has been found that the eosin counterstain lightens to some extent the hæmatoxylin stain of the gelatin. In this case the alum differentiation does not improve the final result, as the gelatin then stains somewhat with the eosin; this eosin staining of the gelatin is less convenient than the hæmatoxylin staining, as most tissue fluids normally take the eosin stain rather than the hæmatoxylin, and can thus be differentiated from any gelatin which may appear in the substance of the section.

The fat stains such as Sudan III. and Scharlach $R$ do not stain the gelatin at all. Staining with these and counterstaining lightly with Mayer's hæmalum gives a clear picture of the fat distribution.

Nile-blue sulphate $A$, as described by Lorrain Smith, is a useful stain, as an excellent differentiation can be obtained by means of 1 per cent. acetic acid. The gelatin becomes completely colourless. 
Weigert's elastic stain also can be used,- - the gelatin stains to some extent with this.

Such stains as Van Gieson's stain, with which it is necessary to pass through alcohols to balsam, are not convenient, as the gelatin shrinks much more than the tissue, and therefore the section becomes very crumpled. Results can be obtained by clipping away the gelatin from the edges of the section, and then staining and taking through to balsam. The gelatin stains very deeply with the stain, and this is also inconvenient. The method is therefore mainly applicable to staining procedures in which no alcoholic stains are used, and alcohol is to be avoided if possible.

The stained sections are floated on to a slide and mounted in some watery medium.

The mounting medium that is on the whole most convenient is glycerin jelly made as follows: gelatin is soaked thoroughly in cold water, drained and then melted on a water bath. When melted, glycerin is incorporated in the proportion of 2 parts to 3 of gelatin, the whole is thoroughly mixed and then cleared with white of egg. A crystal of thymol is added to prevent the growth of moulds. This mixture solidifies at room temperature, and can be kept for months; when wanted it is melted up on a water bath. The medium has the advantage that no ringing of the cover-glass is necessary, and also that it sets in a few seconds. It has one drawback, in that a thin film of jelly tends to cover the upper surface of the cover-glass, and moisture condenses on this film. The slide, unless freshly made, has therefore to be wiped dry before being examined. This drawback can be removed by ringing the cover-glass; the upper surface of it can then be thoroughly cleaned. Other media such as Farrant's solution or lævulose syrup can also be used.

In conclusiou, the method is chiefly of value for very small objects, loose tissue such as the lung, and such organs as the spleen and pancreas which disintegrate easily. It has also been used with success for obtaining complete sections of invertebrates.

By the above method it has been possible to show that the plasmolysis and vacuolation of nerve cells in the cord which has been described in acute anterior poliomyelitis is really a fatty degeneration of the nerve cell. In tetanus also, fatty changes have been observed in the nerve cells. Investigations on these lines are being undertaken into the general question of the so-called vacuolation of the degenerating nerve cell.

Investigations are also in progress by this method into the histological changes in broncho-pneumonia, and in the choroid plexus in various cerebral diseases. In investigations in invertebrates, the method has been used with success for the examination of members of the Hirudineæ, Polychætes and Oligochætes. It has also been found useful in investigating the distribution of the tissue 
corresponding to the suprarenal medulla in Petromyzon fluviatilis $\left(1912^{2}\right)$.

I desire to express my deep indebtedness to Dr. R. Cattley of Leeds, who first introduced me to methods of gelatin embedding, and to Professor Aschoff for advice on many points in the process of cutting and treating frozen sections.

\section{REEERENCES.}

1. Hamilton, D. J. . . . . "Text-book of Pathology," London, 1889, vol. i.

2. Gaskell, J. F. . . . . Journ. Physiol., London, 1912, vol. xliv. p. 59.

3. GayLord aNd Aschoff . "Course of Pathological Histology," Philadelphia, 1901, p. 29. 